\title{
Extensive Void Defects in Mesoporous Aluminosilicate MCM-41
}

\author{
Hong-Ping Lin, ${ }^{\dagger}$ She-Tin Wong, ${ }^{\ddagger}$ Chung-Yuan Mou, ${ }^{*}$, and Chih-Yuan Tang $\$$ \\ Institute of Atomic and Molecular Sciences, Academica Sinica, P.O. Box 23-166, Taipei, Taiwan, 106, and \\ Departments of Chemistry and Zoology, National Taiwan University, Taipei, Taiwan, 106
}

Received: April 25, 2000; In Final Form: June 22, 2000

\begin{abstract}
Mesoporous MCM-41 materials with a distinct $\mathrm{N}_{2}$-sorption hysteresis behavior have been prepared from pure silica and aluminosilicate $-\mathrm{C}_{16}$ trimethylammonium (TMA)Br systems by a delayed neutralization procedure. On the basis of the analysis of transmission electron microscopy micrographs of these MCM-41 materials, we observed that the sample with large type-H4 hysteresis loop at $p / p_{0}$ between 0.5 and 1.0 contains extensive structural defect holes amid the nanochannels. These holes are irregular in shape and their size distributes between 5.0 and $30.0 \mathrm{~nm}$. The pore-blocking effect leads to the hysteresis in desorption. Aluminosilicate MCM-41 often possesses a larger hysteresis loop than pure silica MCM-41. The linear channel system of MCM-41 becomes effectively interconnected through these defect holes. The unusual adsorption hysteresis is associated with the pore-blocking effect around the embedded voids in the framework structures. The size of the adsorption-desorption hysteresis loop is proportional to the volume of hole defects in the nanochannels, and it is dependent on the synthesis conditions such as water content, $\mathrm{Si} / \mathrm{Al}$ ratio, and morphology. Tubular morphology is often associated with large hysteresis behavior and thus more hole defects. The interconnecting channels through defect holes thus makes the diffusion of molecules inside the MCM-41 structure more effective, which is important in catalysis applications.
\end{abstract}

\section{Introduction}

Since their discovery, ${ }^{1}$ the mesoporous silica MCM-41 materials have been extensively investigated for numerous applications such as catalyst support, adsorbent, and electronic and optical devices. ${ }^{2-4}$ In particular, they have shown great potential in catalysis and adsorption because of their large surface area $\left(\sim 1000 \mathrm{~m}^{2} / \mathrm{g}\right)$ and tunable pore size $(1.5-20.0 \mathrm{~nm})$. Their pore size is larger than that of the typical zeolite, and thus MCM-41 can allow bigger molecules in the pore..$^{5-8}$

According to the results of many previous investigations, ${ }^{9-12}$ the structure of these mesoporous MCM-41 materials usually consists of uniform one-dimensional nanochannels. However, the highly ordered one-dimensional MCM-41 channels would make the interchannel transportation ineffective, especially for larger molecules. Thus, mesoporous systems with more effective interchannel accessibility are interesting candidates for many applications. They allow faster mass transport for reactants or products in catalytic reactions and in adsorption of bigger molecules. ${ }^{9}$ These materials are particularly suitable for industrially important catalytic reactions such as dehydrogenation, hydrocracking, and hydrodesulfurization, where catalyst deactivation by coking is a major problem. ${ }^{13}$ To extend the application of these mesoporous materials, it is highly desirable to find a way to improve the accessibility of MCM-41 channel systems.

MCM-48, which has a bicontinuous three-dimensional network structure, would be a good candidate for catalyst or catalyst support because of the high accessibility of its mesoporous channel system. ${ }^{14}$ However, owing to its low hydrothermal and

* Author for correspondence. Fax: 886-2-2366-0954; e-mail: cymou@ ccms.ntu.edu.tw.

Institute of Atomic and Molecular Sciences.

$\doteqdot$ Department of Chemistry.

$\S$ Department of Zoology. thermal stability, the application of MCM-48 is restricted. Recently, a family of mesoporous molecular sieves with stable framework structure (denoted as MSU-G) has been successfully synthesized. ${ }^{15,16}$ MSU-G can have either a vesiclelike hierarchical structure, a wormlike mesoporous structure, or a bicontinuous nanoporous silica structure. Both the methods for preparing a molecular sieve with bimodal pore system and bicontinuous network structure were also provided. The former allows better mass transport within its framework structure (large pore system) and possesses high surface area (small pore system). It was proposed that mesoporous materials with high porosity could be obtained by varying the synthesis procedure and gel composition. For wormhole silica framework (denoted as HMS), it was previously found that textural mesoporosity could be controlled and their catalytic activity in some liquid phase reaction is greatly dependent on the textural porosity for transporting the reagent to the pore sites. ${ }^{17}$ However, these HMS materials are made, using neutral surfactant, in acid synthesis conditions, ${ }^{18}$ which leads to smaller particles ${ }^{19}$ and thus larger interparticle porosity. The acid-made materials are usually less stable, thermally and hydrothermally, or the smallness of particles is not convenient in applications.

The MCM-41 materials made in alkaline condition are, however, more stable and of larger grain size. Postsynthesis treatments can further improve their stability to a higher degree. ${ }^{20,21}$ The remaining problem of large-sized particulate MCM-41 in catalytic applications seems to be its difficulties in transporting reagents. Thus, it would be desirable to fabricate MCM-41 with interchannel connections. It is the purpose of this paper to show that better interchannel connections can be achieved in MCM-41 by creating a large amount of defect cavities in the channel system. We shall discuss the synthesis method, the evidence, and its origin.

Previously, we have provided a convenient "delayed neutralization" method for the alkaline synthesis of pure silica and 
aluminosilicate MCM-41.22 Besides, large-sized TWT (tubulewithin-tubule) hierarchical structure could be obtained through a gradual acidification process. ${ }^{23,24} \mathrm{Here}$, we found that MCM41 prepared from the gel compositions has a special type of $\mathrm{N}_{2}$-sorption isotherms. ${ }^{25}$ There exists an unusual type- $\mathrm{H} 4$ hysteresis loop (according to IUPAC terminology), especially in the case of aluminosilicate MCM-41. The origin of this hysteresis loop was not clearly identified previously. In this paper, we shall show detailed transmission electron microscopy (TEM) micrographs and the adsorption-desorption isotherms to understand the unusual behavior.

We have previously studied a series of molybdenum oxide and iron oxide catalysts supported on this defect-embedded MCM-41 and the catalyzed ethylbenzene dehydrogenation reaction. ${ }^{26}$ The performance of catalyst with support of different morphology was examined. When TWT MCM-41 with a large type-H4 hysteresis loop was used as the catalyst support, the catalytic activity was the highest. We have explained the enhanced activity of this catalyst by correlating the type- $\mathrm{H} 4$ hysteresis loop with the formation of structural defects in the TWT MCM-41 framework. These defects will cause interchannel connection and hence facilitate the diffusion of reactant and product in the catalyst. At the same time, we did not provide adequate characterization results to reveal the true nature of these defects. Besides, the controlling factors of MCM-41 synthesis on the property of defects were not provided. In this paper, we followed on our previous study but focused mainly on the physical nature of these defects.

In this paper, we shall provide the $\mathrm{N}_{2}$ adsorption-desorption and microtome TEM results to further support our proposal that the type-H4 hysteresis loop is attributed to defects in the framework of MCM-41. Furthermore, we have examined the controlling factors of MCM-41 synthesis, including water content and $\mathrm{Al} / \mathrm{Si}$ ratio, on the size of this hysteresis loop. In addition, the amount of structural defects is also closely related to the morphology of MCM-41. Especially for the TWT morphology, the nanochannels of MCM-41 become effectively interconnected and could provide better transport of large molecules in the framework structure.

\section{Experimental Section}

2.1. Materials. The silica source is sodium silicate $\left(27 \% \mathrm{SiO}_{2}\right.$, $14 \% \mathrm{NaOH}$ ) from Aldrich, and the source of aluminum is sodium aluminate $\left(\mathrm{NaAlO}_{2}\right)$ from Riedel-de Haën. The templating agents are alkyltrimethylammonium halides $\left[\mathrm{C}_{n} \mathrm{H}_{2 n+1^{-}}\right.$ $\left(\mathrm{CH}_{3}\right)_{3} \mathrm{NX} ; n=10-16$ and $\mathrm{C}_{n} \mathrm{TMAX} ; \mathrm{X}=\mathrm{Cl}$ or $\left.\mathrm{Br}\right]$ purchased from either Tokyo Chemical Industry or Acrôs. Sulfuric acid $\left(\mathrm{H}_{2} \mathrm{SO}_{4} ; 96 \%\right)$ is from Merck. All these chemicals were used directly without further purification.

2.2. Synthesis. The typical synthesis procedure for preparing pure silica and aluminosilicate MCM- 41 is based on the delayedneutralization process reported in our previous paper. ${ }^{22,23}$ To prepare pure-silica MCM-41 materials, sodium silicate was added to a clear aqueous solution of the surfactant under stirring and a gel mixture was formed. After stirring for about $10 \mathrm{~min}$ at room temperature, a proper amount of $1.10 \mathrm{M}$ sulfuric acid was added into the gel mixture with a pipette. This step takes up to $30 \mathrm{~min}$. The $\mathrm{pH}$ value of the final mixture was adjusted to about 10. Generally, the molar composition of the resultant gel to prepare the well-ordered MCM-41 materials is: $1.0 \mathrm{C}_{n^{-}}$ TMAX: $2.10 \mathrm{SiO}_{2}:(0-0.21) \mathrm{NaAlO}_{2}:(1.63-1.20) \mathrm{NaOH}$ : (0.67-0.40) $\mathrm{H}_{2} \mathrm{SO}_{4}$ : (50-500) $\mathrm{H}_{2} \mathrm{O}$. However, TWT products can be obtained only in a narrow range of water content, $\mathrm{Si} / \mathrm{Al}$ ratio, temperature, or upon the addition of 1-alkanols. The
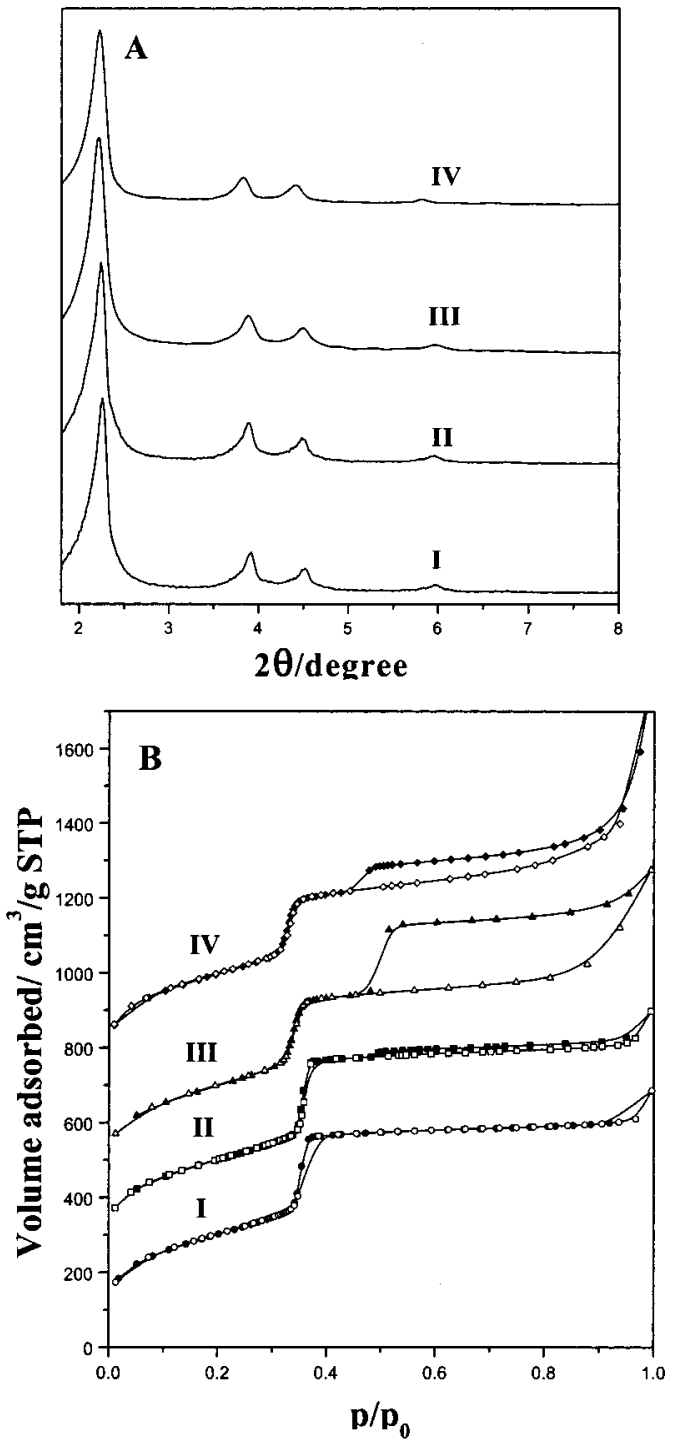

Figure 1. XRD patterns (A) and $\mathrm{N}_{2}$ adsorption-desorption isotherms (B) of MCM-41. I: $\mathrm{Si} / \mathrm{Al}=\infty$, water $/ \mathrm{C}_{16} \mathrm{TMABr}=352$ (particle); II: $\mathrm{Si} / \mathrm{Al}=\infty$, water $/ \mathrm{C}_{16} \mathrm{TMABr}=225$ (particle); III: $\mathrm{Si} / \mathrm{Al}=37$, water/ $\mathrm{C}_{16} \mathrm{TMABr}=168$ (TWT); IV: $\mathrm{Si} / \mathrm{Al}=37$, water/ $\mathrm{C}_{16} \mathrm{TMABr}=279$ (particle).

detailed synthetic procedures and components can be found elsewhere. ${ }^{23,24}$ The resultant gel mixture was hydrothermally reacted at $100{ }^{\circ} \mathrm{C}$ for $48 \mathrm{~h}$ in a static autoclave. The assynthesized product was recovered by filtration and washed thoroughly with deionized water. The dried product was then calcined at $560{ }^{\circ} \mathrm{C}$ in air for $6 \mathrm{~h}$ to remove the template.

2.3. Characterization. The powdered $\mathrm{X}$-ray diffraction (XRD) patterns of the as-synthesized and calcined samples were collected on a Scintag X1 diffractometer using $\mathrm{Cu} \mathrm{K \alpha}(\lambda=$ $0.154 \mathrm{~nm}$ ) radiation. $\mathrm{N}_{2}$ adsorption-desorption isotherms of the calcined samples were obtained on a Micromeritics ASAP 2010 system at $77 \mathrm{~K}$. The samples were outgassed at $250{ }^{\circ} \mathrm{C}$ under a pressure of $10^{-3}$ Torr for at least $3 \mathrm{~h}$. Bulk mesoporous materials for TEM characterization were embedded in Spur resin and cured at $60{ }^{\circ} \mathrm{C}$ overnight. Ultrathin sections (approximately $60-90 \mathrm{~nm}$ ) were cut from the embedded specimen using a diamond knife at room temperature. They were laid on copper grids. TEM micrographs were taken with a Hitachi H-7100 instrument operated at $75-100 \mathrm{keV}$. Scanning electron microscopy (SEM) micrographs were taken with a Hitachi S-800 instrument operated at an accelerating voltage of $20 \mathrm{keV}$. The 

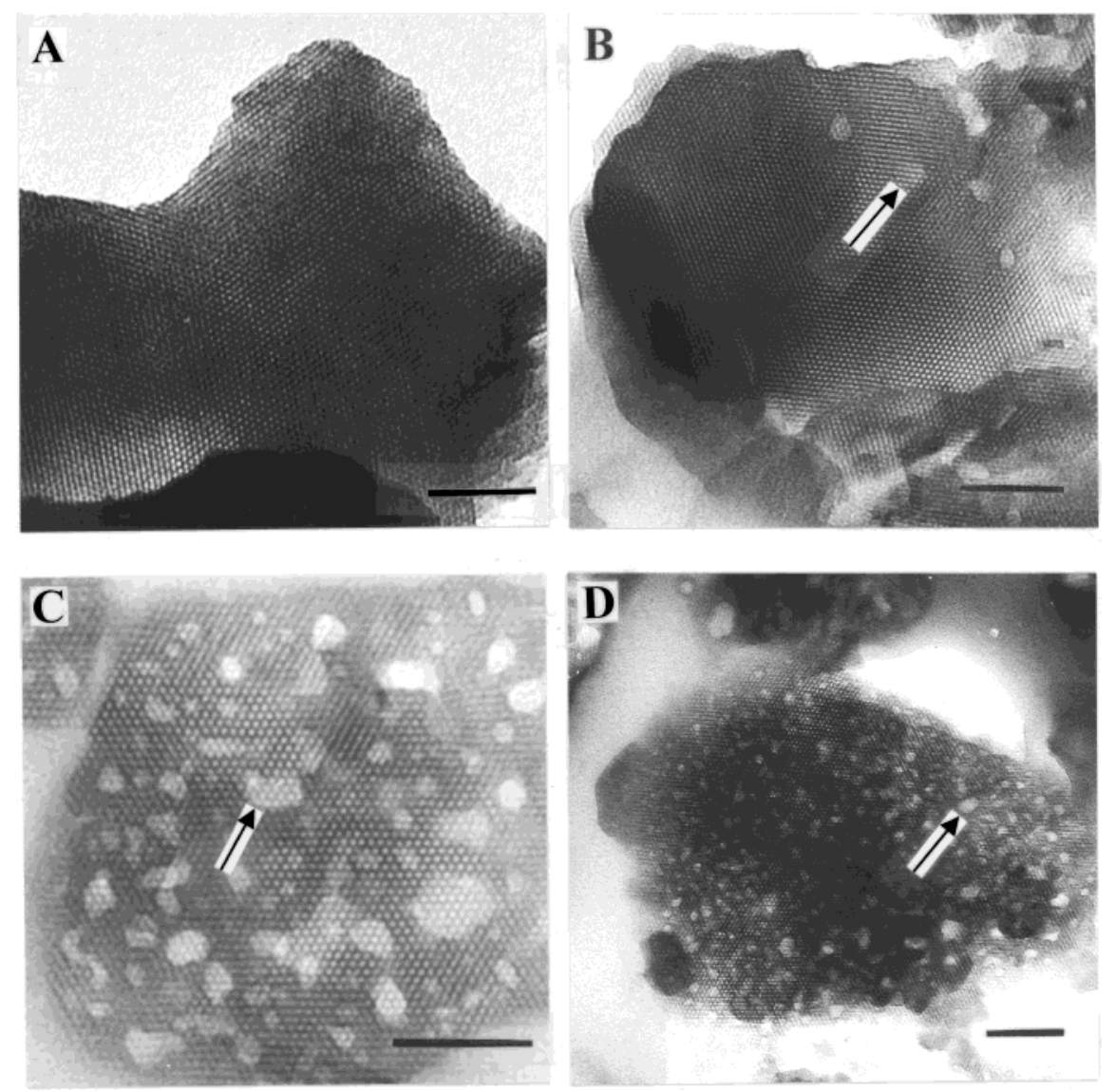

Figure 2. Top-view microtome TEM micrographs on thin sections of MCM-41 samples shown in Figure 1. A, sample I; B. sample II; C. sample III; D, sample IV. Scale bar $=77 \mathrm{~nm}$.

solid state ${ }^{29} \mathrm{Si}$ or ${ }^{27} \mathrm{Al}$ magic-angle spinning (MAS) NMR experiments were performed at room temperature on a Bruker MSL 300 or 500 NMR spectrometer with a magnetic field of 6.6 or $11 \mathrm{~T}$, respectively.

\section{Results}

3.1. Adsorption Hysteresis in MCM-41. Figure 1A shows the XRD patterns of the calcined MCM-41 samples synthesized from $\mathrm{C}_{16} \mathrm{TMABr}$-silicate (samples I and II are pure silica MCM-41 samples in particulate form) and aluminosilicate compositions (samples III and IV are aluminosilicate MCM-41 materials of $\mathrm{Si} / \mathrm{Al}=37$; sample III is in TWT morphology, but sample IV is in microparticle form) at various water content (as listed in figure caption) by using the delayed neutralization process. There exist at least four sharp XRD peaks for all these samples, indicating well-ordered hexagonal structure of MCM41. These results are consistent with previously reported results that the incorporation of aluminum into silica framework does not have a significant effect on the ordering of mesostructure. When examining the $\mathrm{N}_{2}$ adsorption-desorption isotherms of these samples (Figure 1B), one can clearly see that a sharp inflection that is common to all these samples exists at $p / p_{0}=$ 0.32 . This inflection is typical of a capillary condensation process and the $p / p_{0}$ value corresponds to a pore size of about $2.6 \mathrm{~nm}$ (calculated from the Barrett-Joyner-Halenda method). The pore size distribution of these samples is also narrow, and the full width at half-maximum is only $0.12-0.14 \mathrm{~nm} .{ }^{27}$ However, samples II, III, and IV possess an additional uncommon type-H4 hysteresis loop at $p / p_{0}$ between 0.5 and 1 . The assignment of hysteresis is made according to de Boer's classification. Here, we define the size of the hysteresis loop in the isotherm as the difference in $\mathrm{N}_{2}$ adsorption volume between the desorption and adsorption branches in the range stated above. The size of the hysteresis loop decreases in the order: sample III $\left(150 \mathrm{~cm}^{3} / \mathrm{g} \mathrm{STP}\right)>$ IV $\left(50 \mathrm{~cm}^{3} / \mathrm{g} \mathrm{STP}\right)>$ II $\left(16 \mathrm{~cm}^{3} / \mathrm{g} \mathrm{STP}\right)$ $>$ I $\left(0 \mathrm{~cm}^{3} / \mathrm{g}\right.$ STP $)$. The existence of such a type-H4 hysteresis loop might indicate that some structural defects are formed in the MCM-41 hexagonal channel matrix.

A similar type-H4 hysteresis loop that does not exhibit any limiting adsorption at high $p / p_{0}$ values has been observed in a sample having aggregates of platelike particles, leading to a slitshaped pore structure. ${ }^{28,29}$ We shall discuss our interpretation of these special hysteresis in section 4. In this section, we first report the empirical correlation of adsorption hysteresis with TEM micrographs and synthesis conditions.

3.2. Adsorption Hysteresis and Structural Defects in MCM-41. Different types of adsorption hysteresis exist in mesoporous materials. Up to the present, most of the adsorption hysteresis reported is capillary hysteresis; ${ }^{30,31}$ for example, the vertical capillary condensation and evaporation do not occur at the same vapor pressure. This happens only for pore size greater than $4 \mathrm{~nm}$. The hysteresis is associated with metastability of the adsorption branch of the isotherm. ${ }^{32}$ Because the pore size in our sample is less than $4 \mathrm{~nm}$, this capillary hysteresis does not occur in our materials.

The hysteresis we observed is in fact extraordinarily large and rarely observed in MCM-41. In the literature, the type-H4 hysteresis loop has been ascribed to a slitlike pore structure that is commonly observed in the pillared materials. ${ }^{26,27} \mathrm{We}$ would like to show in this section that the type of hysteresis we observed can be correlated with a specific pore defect. To understand further the relation between hysteresis and pore 


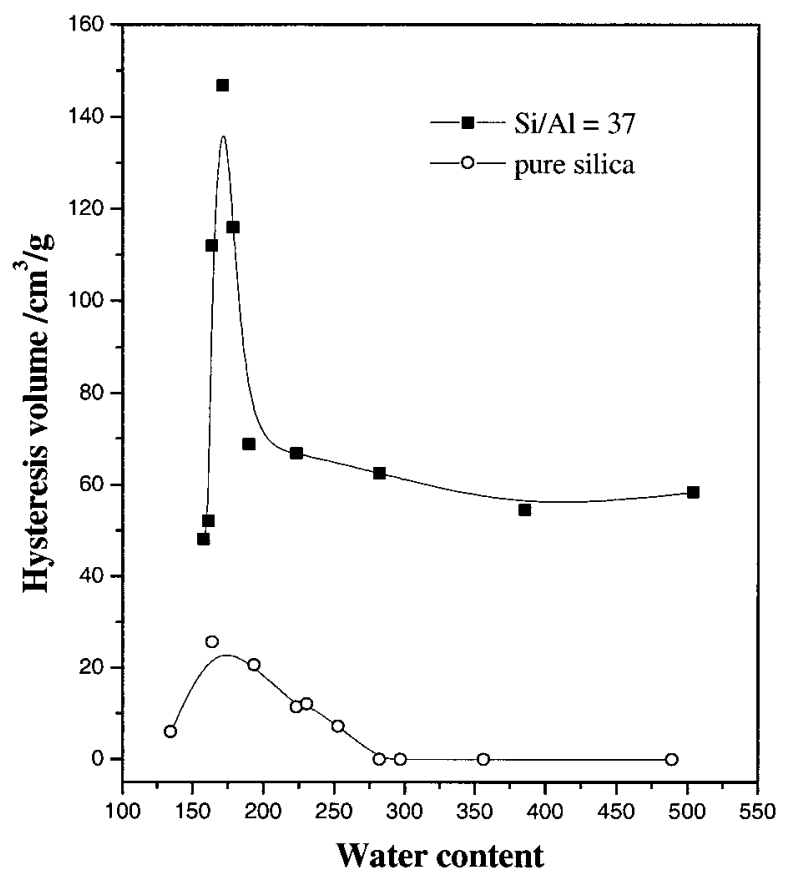

Figure 3. Plot of hysteresis loop size of pure silica and aluminosilicate $(\mathrm{Si} / \mathrm{Al}=37) \mathrm{MCM}-41$ versus water content.

structure of MCM-41, a microtome TEM technique was used. In the microtomed TEM micrographs on ultrathin sections of these samples (Figure 2), we can see clearly the characteristic honeycomblike MCM-41 hexagonal structures. However, one can also find additional voids interspersed extensively in samples II, III, and IV (shown by arrows). These voids (white regions) are attributed to structural defects. The size of these defects is not uniform (distributes between 5.0 and $30.0 \mathrm{~nm}$ ) and their shapes are irregular. Moreover, the amount and size of the structural defects in MCM-41 increase in the order: III > IV > II $>$ I. This sequence is parallel to the size of the hysteresis loop. In other words, the sample with a larger hysteresis loop has more structural defects. Thus, we can conclude that the large type-H4 hysteresis loop in MCM-41 originates from these structural defects, rather than from the slit or lamellar type of pore structure as previously reported. Indeed, microtome TEM technique is a powerful tool. It allows us to observe directly the bulk structure of MCM-41, rather than the external morphology as in the case of high-resolution SEM. ${ }^{33}$ The defective MCM-41(def-MCM) framework can be considered as a bundle of short and discontinuous pure silica or aluminosilicate nanochannels. An MCM-41 sample consisting of shorter nanochannels possesses a larger hysteresis loop.

We have found in the ${ }^{27} \mathrm{Al}$ and ${ }^{29} \mathrm{Si}$ MAS NMR spectra (not shown here) of the as-synthesized and calcined MCM-41 materials exhibited in Figures 1 and 2 that the coordination and degree of local order of aluminum and silica are similar to those reported previously. ${ }^{34-36}$ Thus their microscopic structure is normal. The delayed neutralization procedure is mainly responsible for creating the void defect in the MCM-41 materials reported here.

3.3. Effect of Synthesis Conditions on Size of Hysteresis Loop. From Figure 1, one can see that pure silica and aluminosilicate MCM-41 synthesized from different water content have distinctively different hysteresis loop size. Besides, aluminosilicate MCM-41 possesses a larger hysteresis loop than that of pure silica MCM-41 under similar water content. These results clearly indicate that the size of the hysteresis loop is related to both water content and the amount of $\mathrm{Al}$ incorporated
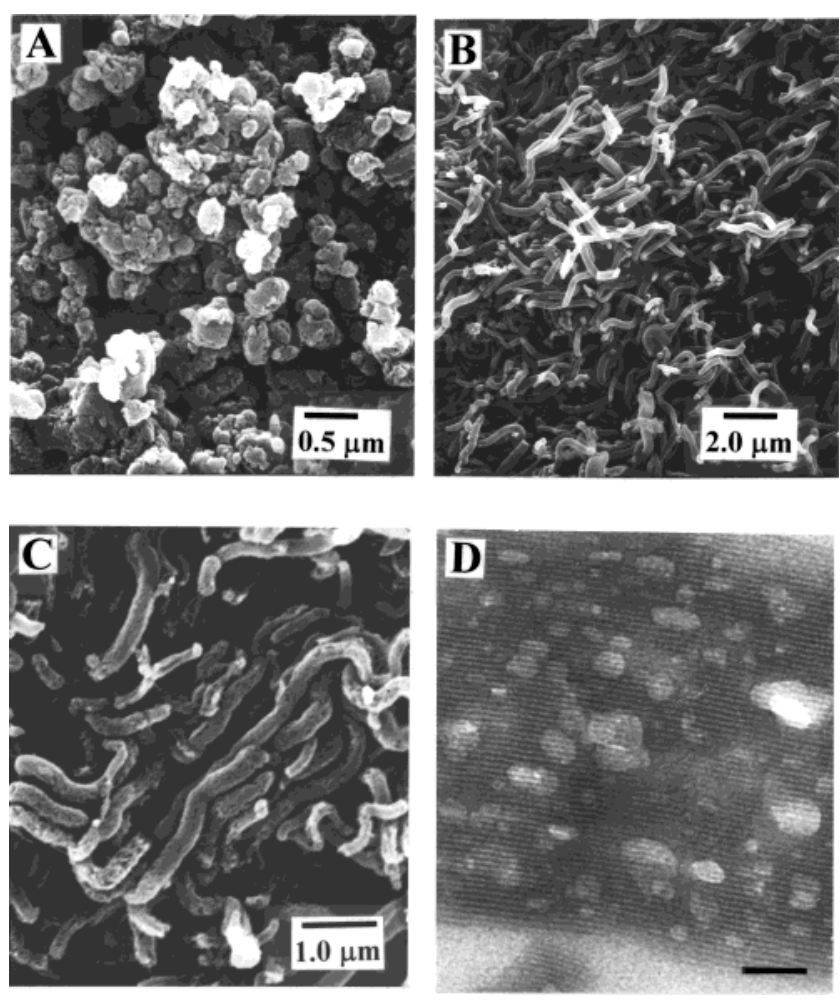

Figure 4. SEM and TEM micrographs of aluminosilicate $(\mathrm{Si} / \mathrm{Al}=$ 37) MCM-41 synthesized with different water content. (A) SEM showing particle morphology, water $/ \mathrm{C}_{16} \mathrm{TMABr}=279$; (B) SEM showing TWT morphology, water/ $\mathrm{C}_{16} \mathrm{TMABr}=168$; (C) larger magnification of B; (D) TEM of B (scale bar $=45 \mathrm{~nm}$ ).

in the silica framework. Thus, we must further discuss these two synthesis factors to offer an optimum composition for preparing MCM-41 with the desired amount of structural defects.

Figure 3 shows that varying the water content has a remarkable influence on the hysteresis loop size of pure silica and aluminosilicate $(\mathrm{Si} / \mathrm{Al}=37) \mathrm{MCM}-41$. First, the size of the hysteresis loop for aluminosilicate MCM-41 is always larger than that of pure silica under the same water content. This means that aluminum incorporation into the silica framework would promote the formation of structural defects. According to Lowenstein's rule, crystalline aluminosilicates such as zeolites generally avoid the formation of $\mathrm{Al}-\mathrm{O}-\mathrm{Al}$ moieties in their framework. The crystal size of MCM-41 is reduced by the incorporation of aluminum into the silica framework. This structural factor makes the nanochannels of aluminosilicate MCM-41 shorter than those of silica MCM-41. The shorter nanorods of the former in the synthesis medium then restructure into a more defective framework (i.e., def-MCM-41; Figure 2C and D), whereas those of the latter arrange into a less defective or a perfect framework (Figure $2 \mathrm{~A}$ and $\mathrm{B}$ ).

Second, there is a sharp rise in the hysteresis loop size within a narrow range of water content in both pure silica and aluminosilicate MCM-41. To understand this result, we examined the SEM micrographs of aluminosilicate MCM-41 synthesized from water content of 279 and 168, and having hysteresis loop size of $68 \mathrm{~cm}^{3} / \mathrm{g}$ and $146 \mathrm{~cm}^{3} / \mathrm{g}$ at STP, respectively (Figure $4 \mathrm{~A}$ and $\mathrm{B}$ ). The sample in Figure $4 \mathrm{~A}$ is mostly in the normal microparticle morphology, whereas that of Figure 4B is nearly in the TWT morphology. Under larger magnification, the TWT hierarchical structure is clearly revealed in the SEM (Figure 4C) and TEM (Figure 4D) micrographs. More importantly, there are a variety of structural defects in 


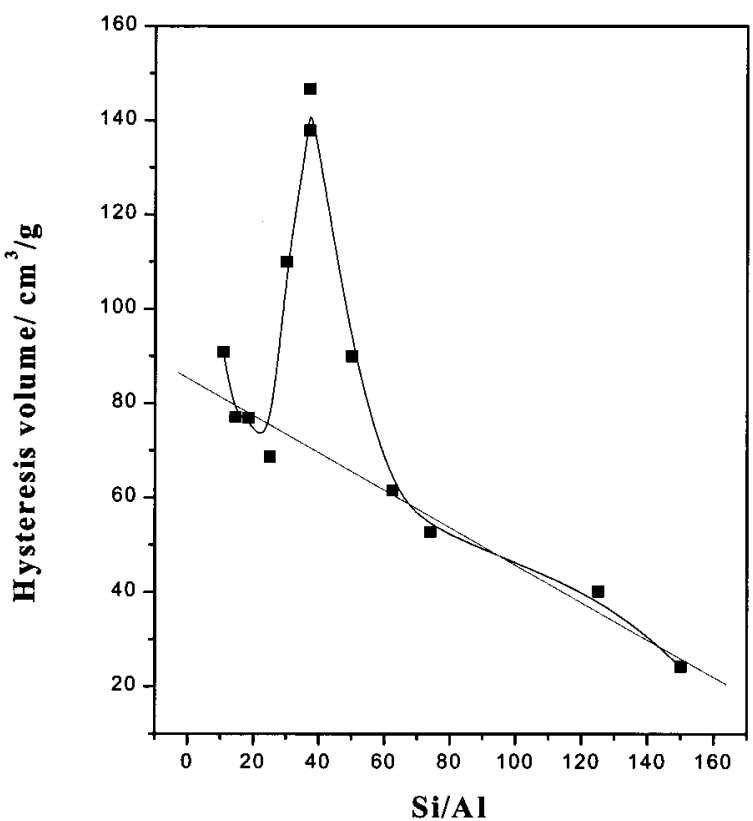

Figure 5. Plot of hysteresis loop size of aluminosilicate MCM-41 versus $\mathrm{Si} / \mathrm{Al}$ ratio at water $/ \mathrm{C}_{16} \mathrm{TMABr}=168$.

the framework system. Thus for aluminosilicate MCM-41, TWT morphology always leads to a larger hysteresis loop and more structural defects than particulate morphology. Therefore, the
TWT morphology of MCM-41 is also an indicator for the formation of structural defects that are responsible for the creation of the hysteresis loop.

The influence of water content on the size of the hysteresis loop can be understood by the mechanism of TWT formation outlined in our previous report. ${ }^{23}$ The TWT hierarchical structure was assumed to be formed from surfactant-aluminosilicate liquid crystal where the hexagonal and lamellar phases coexist. Thus there is a narrow range of surfactant/water ratio for the TWT. For the surfactant-silicate system, it was found that the water content is crucial for TWT formation. ${ }^{24}$ The SEM pictures of the MCM-41 materials synthesized from $\mathrm{C}_{12} \mathrm{TMAB}-\mathrm{C}_{4} \mathrm{OH}-$ silicate with different water content show that tubular morphology could be formed only in a proper water content range. At low water content, the sample is in microparticle form even when the $\mathrm{C}_{m} \mathrm{OH}$ concentration was varied. ${ }^{29}$ In our previous reports of TWT, ${ }^{23}$ it was proposed that a mixed lamellarhexagonal membrane intermediate, separated by water layers, is involved. The membranes are stabilized by the electrostatic and entropy undulation repulsion forces between the membranes. At low water content condition, the membranes are tightly aggregated and would not have adequate energy for curving them into TWT morphology. With higher water content, the lamellar-hexagonal layers are separated by water and they are soft enough to be curled to TWT. ${ }^{23}$ Therefore, the narrow range of water content where both phases coexist is essential for TWT formation. When further silica condensation happens, the extra
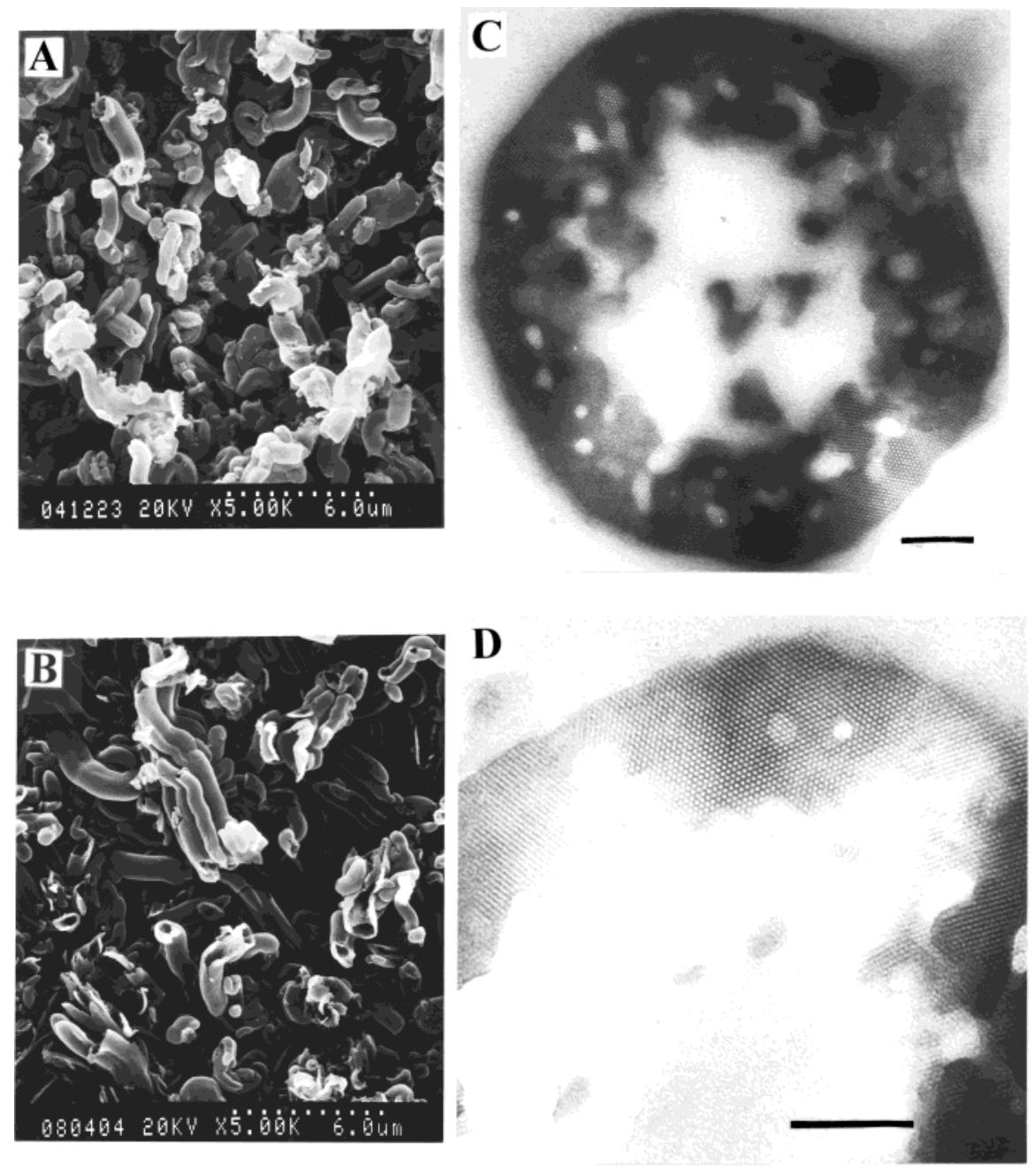

Figure 6. SEM and microtome TEM micrographs of hollow pure-silica TWT MCM-41 synthesized from $\mathrm{C}_{16} \mathrm{TMACl}^{-}$silicate $(\mathrm{A}, \mathrm{C})$ and $\mathrm{C}_{14-}$ TMABr-BuOH-silicate (B, D) compositions. Scale bar in Figure $6 \mathrm{C}$, D is $85 \mathrm{~nm}$. 
interlayer water is expulsed to form little puddles. Consequently, we have many voids formed as the relic of water puddles. ${ }^{37,38}$

This is the reason MCM-41 samples synthesized from such a water range have a larger hysteresis loop size (Figure 3). Although pure silica MCM-41 with TWT morphology can also be prepared from $\mathrm{C}_{16} \mathrm{TMABr}$ - silicate composition at a proper water content, the TWT yield is low (about 10\%). Probably, because of the lower yield, its maximum hysteresis loop size is smaller than that of aluminosilicate MCM-41. Further works are needed to understand why the structural defects prefer to grow in the sample with TWT morphology. In brief, the formation of structural defects in the framework of MCM-41 depends mainly on the amount of incorporated aluminum and water content, which is reflected in sample morphology.

Next, we shall show that the amount of structural defects increases with aluminum content in aluminosilicate MCM-41. Figure 5 shows the variation of hysteresis loop size of aluminosilicate MCM-41 synthesized with the same water content of 168 , but different $\mathrm{Si} / \mathrm{Al}$ ratios. Generally, the hysteresis loop size increases linearly with aluminum content, as previously assumed. But a sharp rise in loop size is also observed between $\mathrm{Si} / \mathrm{Al}$ values of 20 and 65 . Referring to the SEM micrographs of these aluminosilicates, one can see that only TWT morphology can be obtained in this $\mathrm{Si} / \mathrm{Al}$ range. Outside this range, microparticles were formed. This phenomenon could be due to the fact that aluminum content also plays an important role for the creation of TWT morphology. ${ }^{24}$ As we have described previously, ${ }^{24}$ aluminum incorporation can reduce the size of the membranelike mesostructure intermediate of $\mathrm{C}_{16} \mathrm{TMABr}$-aluminosilicate. When its size reaches a proper dimension, the membrane can bend into a more thermodynamically stable TWT morphology. At the lower and higher ends of aluminum content, the intermediate is too thick (or small) to create the TWT morphology. Therefore, microparticles were formed instead of TWT. In summary, the amount of defects in particlelike aluminosilicate MCM-41 increases linearly with aluminum content. However, the creation of TWT morphology further enhances the formation of an extra amount of void defect.

3.4. Pore System in Pure Silica Hollow TWT MCM-41. Because tubular morphology is generally associated with extensive void defects, we would like to examine more closely the various tubular forms and the associated defects. In addition to the Al-containing TWT hierarchical structure, ${ }^{23}$ pure silica TWT hierarchical structure can also be synthesized from $\mathrm{C}_{16}$ $\mathrm{TMACl}-$ silicate or $\mathrm{C}_{14} \mathrm{TMABr}-\mathrm{BuOH}$-silicate composition as reported previously.$^{29}$ The two SEM micrographs in Figure 6A and B show that the hollow TWT MCM-41 can be obtained in high yield. The microtome TEM micrographs in Figure $6 \mathrm{C}$ and D offer a better view of these structures, which consist of a micron-sized central pore (indicated by arrow I), submicronsized structural defects (arrow II), and nanochannels. This is the first example of a mesoporous sample with a trimodal pore system, for example, the nanochannels of MCM-41, and the voids and the central big hollow channel (micron-size) of TWT. As in the case of aluminosilicate TWT MCM-41, this hollow pure silica TWT sample also possesses a larger hysteresis loop (about $40-100 \mathrm{~cm}^{3} / \mathrm{g} \mathrm{STP}$ ) than that of particlelike MCM-41 (Figure 7). It is reasonable to suppose that the channels of pure silica TWT MCM-41 are also highly interconnected and could be used as catalyst support or adsorbent for large molecules.

3.5. Varying Surfactant Chain Length. Next, we investigate the hysteresis loop using surfactant with different carbon chain length. Using the same delayed neutralization process, we make the MCM-41 materials at a proper water content where void

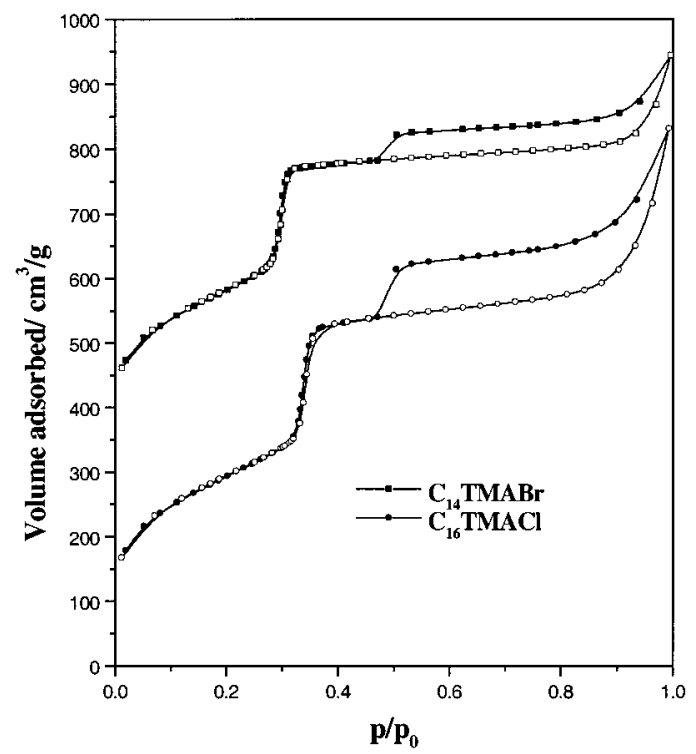

Figure 7. $\mathrm{N}_{2}$ adsorption-desorption isotherms of hollow pure-silica TWT MCM-41 obtained from $\mathrm{C}_{16} \mathrm{TMACl}-$ silicate and $\mathrm{C}_{14} \mathrm{TMABr}-$ $\mathrm{BuOH}-$ silicate compositions.

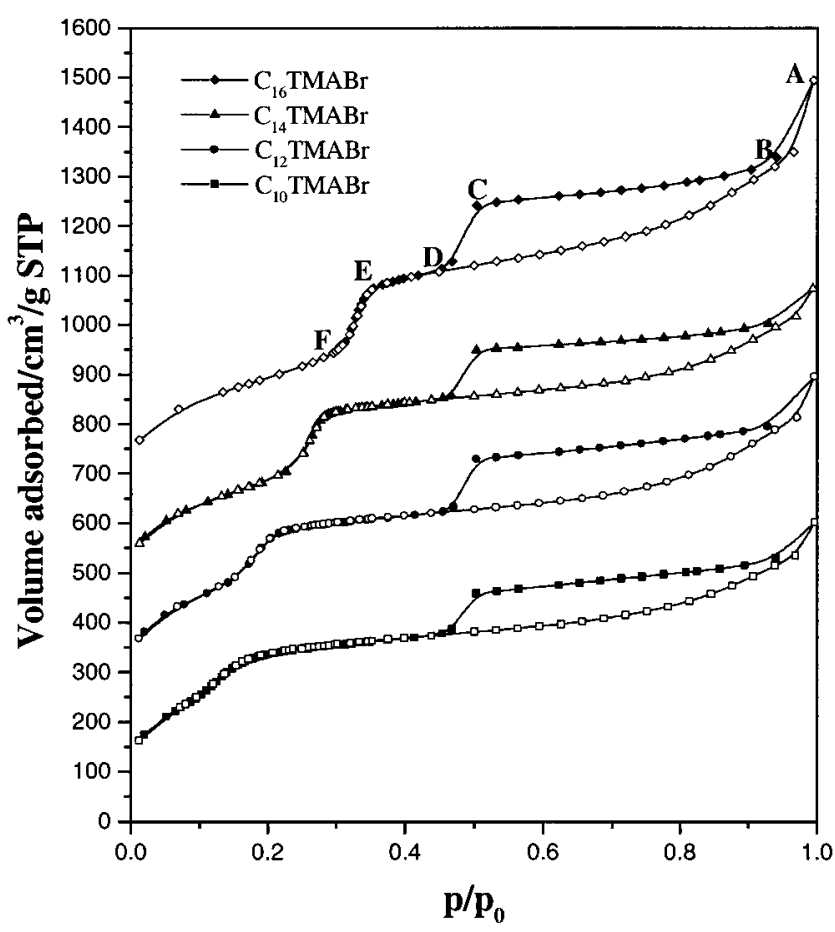

Figure 8. $\mathrm{N}_{2}$ adsorption-desorption isotherms of aluminosilicate MCM-41 prepared from $\mathrm{C}_{n} \mathrm{TMABr}-$ aluminosilicate composition $(n=$ 10-16).

formation is expected. Indeed, we can create similar type-H4 hysteresis in the sorption behavior for MCM-41 prepared with all the carbon chain length surfactants, from $\mathrm{C}_{10}$ TMAB to $\mathrm{C}_{16^{-}}$ TMAB. As expected, capillary condensation occurs at lesser vapor pressure for shorter chain length surfactants, giving narrower pore (Figure 8). But surprisingly, all the desorption loops of Def-MCM41 in Figure 8 occurred at the same $p / p_{0}$ value of about 0.5 irrespective of the chain length. It seems that there is a constant curvature for the nucleation of the desorbing nitrogen. It could reflect a heterogeneous nucleation process dictating either a definite curvature or a definite pore size. This phenomenon needs further investigation.

In summary, one can use the delayed neutralization process to create voids up to several tens of nanometers in MCM-41 
materials with varying channel diameters. The existence of structural defects in aluminosilicate MCM-41 made its channels more interconnected than pure silica MCM-41.

\section{Discussion}

In this section, we discuss three aspects of the observation on the extensive void formation in MCM-41 leading to the interconnection between linear channels. First, we explain the cause of the unusual hysteresis behavior of nitrogen adsorption, such as in Figure 1, on the basis of our detailed observation of voids and its correlation with the extent of hysteresis. Second, we shall discuss the cause of the formation of the void defects. Third, we will summarize our previous observation on the better performance of catalyst with tubular MCM-41 as catalyst support in the dehydrogenation of ethylbenzene. The better diffusion in the channel system of TWT is proposed as the cause.

4.1. Hysteresis Behavior in a Network of Mesopores. Whereas the gas adsorption behavior of MCM-41 has been extensively studied, ${ }^{39,40}$ the special type-H4 hysteresis of MCM41 has not been investigated. This, by itself, deserves careful study in the future. Here, we present a brief interpretation on the basis of our nitrogen adsorption data.

We now examine closely the special adsorption curves (Figures 7 and 8) with large and wide hysteresis loops (above $\left.p / p_{0}=0.5\right)$. Everett ${ }^{41}$ in a review of adsorption hysteresis extensively discussed and gave possible explanations for the existence of such a phenomenon. One case deserving special attention is the hysteresis of an extensively interconnected porous network. Later, Mayagoitia et al. ${ }^{42}$ extensively discussed the adsorption/desorption hysteresis in capillary condensation in a network of mesoporous cavity and connecting "channels".

The porous MCM-41 can be visualized as a connected network of alternated elements, the voids, and the capillary channels. The interaction between the voids and the capillary channels are a little bit complicated. Let us examine the top adsorption isotherm (with alphabet labels) in Figure 8, as it is typical in our sample.

To help us understand the whole sorption-desorption process, we use a scheme used by Janicke et al. ${ }^{36}$ to explain the process in Figure 9. Here, we plot the pore-size distribution schematically including the voids. The void sizes are widely distributed according to our TEM observation. The shaded area represents the extent of pore filling. The letters in Figure 9 correspond to those in Figure 8.

For the ascending (adsorption) isotherm, the first vertical rise in volume reflects the size of nanochannels in capillary condensation. The slow rise from points $\mathrm{E}$ to A represents the gradual condensation of nitrogen in the defects and external surface. Upon descending, the rapid decrease from A to B reflects the macropore of interparticle voids. However, the isotherm between B and C remains flat, more or less. Basically, the failure of liquid nitrogen evaporation at pressure greater than point $\mathrm{C}$ in Figure 8 is due to the pore-blocking effect in the defective voids of MCM-41. Some of the voids are close to the external surface and have less pore-blocking effect. Hence the nitrogen is probably easily desorbed. Thus, we still have a slow decrease from $\mathrm{B}$ to $\mathrm{C}$. At point $\mathrm{C}$, however, the driving force for evaporation is strong enough that a lot of cooperative evaporations happen at the same pressure. This is due to the opening-up of the interconnecting channels between voids. The pore-blocking effect can be visualized as in Figure 9 D. The corresponding pore size at points $\mathrm{C}$ and $\mathrm{D}$ is about $3.7 \mathrm{~nm}$, if one uses hemispherical approximation for the invading interface. An alternative interpretation would be that the wall between a

\section{Adsorption Desorption}

I
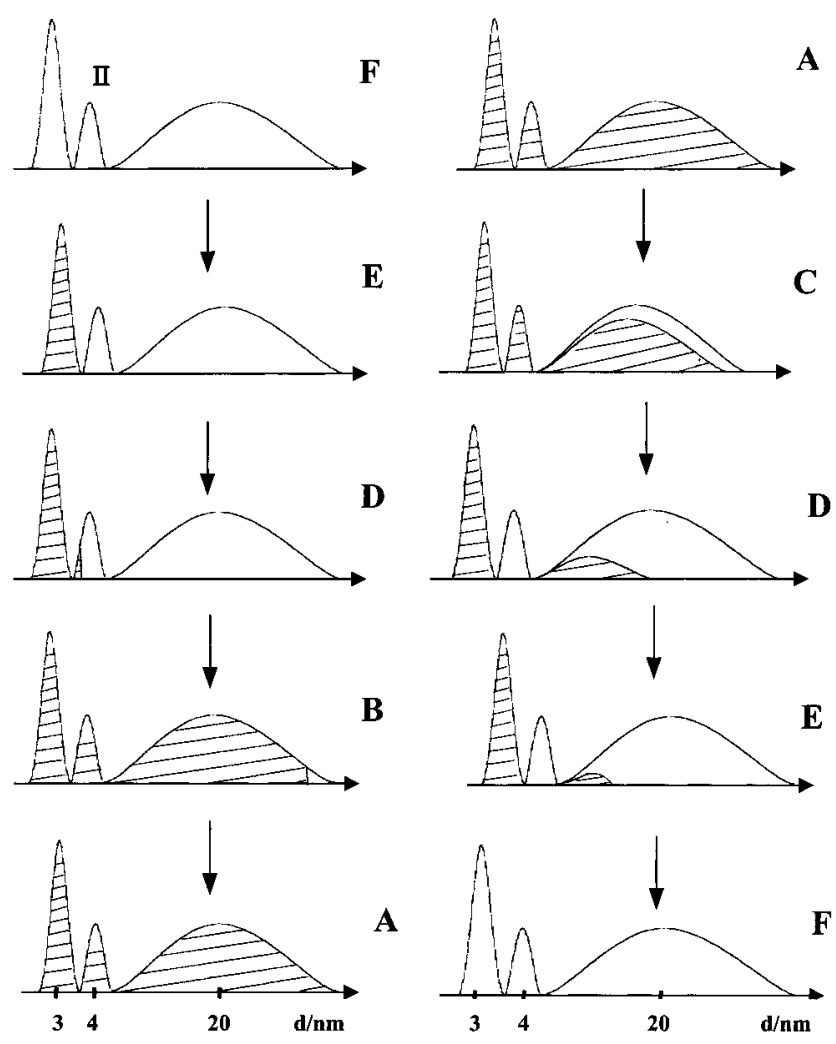

Figure 9. Schematic description of the progress of nitrogen adsorption-desorption in the void containing MCM-41 materials. The letters in this figure denote the stage of evolution as in the top curve of Figure 8. Shaded areas represent the filled portion of the pore size distribution. The sharp left-most peak belongs to the primary pore; the smaller peak represents the defective channels. The broad distribution at large size is due to the internal voids. On the desorption branch, pore blocking leads to the sudden drop between $\mathrm{D}$ and F.

neighboring pair of the void-connecting channels is missing (a line defect) and a hemi-ellipsoidal interface would roughly give one a $p / p_{0}=0.5$ according to the Thomson equation. The TEM micrographs in Figure 2D and Figure 4D could be reasonably interpreted this way. Upon further descending to points $E$ and $\mathrm{F}$, another sharp capillary desorption occurs, and this corresponds to the primary mesopores of MCM-41. Accordingly, one would expect that most of the pore deblocking of the voids would occur between points $\mathrm{C}$ and $\mathrm{D}$ when the hysteresis loop is big. The TEM micrograph of Figure 2D indicates that there are extensive voids in the material, and one expects a higher probability of the void being connected by many defective channels. Thus one has pore deblocking at a pressure higher than the evaporation of the primary pore.

On the basis of hydrogen adsorption studies, Edler et al. ${ }^{43}$ had previously proposed that MCM-41 samples possessing channels interconnected by pathways are capable of supporting bulk hydrogen. Their evidence was a nonequilibrium sudden desorption of hydrogen. The evidence seems similar to ours for nitrogen sorption. However, the cause could be different. Further studies are needed.

4.2. Consequence of Transport in Catalysis. Thus, we have identified for the first time a special void defect structure in MCM-41 by the combination of special synthesis conditions, TEM examination, and nitrogen adsorption study of the hysteresis. The voids are rather densely populated such that individual channels do not run very long before connecting to 
a void. We conclude that the channel network becomes effectively better interconnected because of the existence of voids connecting the channels. One would expect that the transport of materials becomes easier in such defective MCM41. The mixing of molecules across the channels is easier. Such evidence has been previously presented in our MCM-41supported catalysis study. ${ }^{25,26}$ We discuss further the implications in light of the present study.

Previously, we used MCM-41 in both tubular and particulate morphologies as the support for molybdenum and iron oxide catalysts in the ethylbenzene dehydrogenation reaction. ${ }^{26}$ The tubular MCM-41-supported catalyst prepared by physical mixing and spreading gave the highest catalytic activity for both molybdenum and iron oxide in the production of styrene (Figures 5 and 6 of ref 26). We have suggested that the defects formed in MCM-41 made the channels of tubular MCM-41 (denoted as $\mathrm{M}_{\mathrm{T}}$ ) effectively interconnected and provided a better transport of reactant and product in the catalytic reaction. Hence, tubular MCM-41-supported samples gave higher conversion in the catalyzed reaction than particulate MCM-41-supported ones. In light of our observation of extensive voids in the channel system of TWT form of MCM-41, we can interpret the difference in catalytic performance in terms of better material transport in the tubular MCM-41 support.

In the MCM-supported $\mathrm{MoO}_{3}$ catalyst, the tubular MCM-41 supported catalyst $\left(\mathrm{Mo}+\mathrm{M}_{\mathrm{T}}\right)$ is the best-performing catalyst with the highest steady-state and initial activity. ${ }^{26}$ The decay rate is the lowest even under the influence of intense acid-catalyzed side reactions known to produce coke. The high porosity of the tubular framework as a result of interchannel connection played a determining role in the catalytic reaction. The better interchannel diffusion helped catalysis in three ways: (a) Allows a better spreading of $\mathrm{MoO}_{3}$ from external to its entire surface, and this increases the concentration of reactive centers (possibly pairs of neighboring Mo sites). (b) Allows a better diffusion of reactant and product because each segment of the tubule can be considered as a small particle of $M_{P}$. In this respect, the activity will be improved because the diffusion path (to the active site) is shorter than the case with particle morphology. (c) Allows a better spreading of the reduced $\mathrm{MoO}_{\mathrm{x}}$ species formed during the course of reaction through its entire surface, thus lowering the possibility of sintering in a reduced environment. ${ }^{26}$

\section{Conclusions}

The synthesis and characterization of defects, voids, and line defects in silica and aluminosilicate MCM-41 were described. We have provided further information on the fascinating relation between the type-H4 hysteresis behavior and structural defects. The morphology of product formed and the aluminum content in the synthesis gel have apparently affected the amount and nature of the structural defects. These structural defects have the ability of making the nanochannels highly interconnected, and thus, improving the diffusion of large molecules in the MCM-41 framework system. Not surprisingly, we have found that molybdenum oxide or iron oxide supported on TWT aluminosilicate MCM-41 has higher catalytic activity for ethylbenzene dehydrogenation than that on particlelike pure silica MCM-41. Owing to their large surface area and tunable interconnected nanopore size, these mesoporous materials with nanometer size voids in the pore systems can find broad applications in areas such as catalysis, separation technology, biomaterial engineering, and optical, electronic, and sensing devices.
Acknowledgment. This research was supported by the China Petroleum Co. and the National Science Council of Taiwan (NSC 88-2113-M-002-027).

\section{References and Notes}

(1) Kresge, C. T.; Leonowicz, M. E.; Roth, W. J.; Vartuli, J. C.; Beck, J. S. Nature 1992, 359, 710 .

(2) (a) Coma, A. Chem. Rev. 1997, 97, 2373. (b) Wu, C. C.; Bein, T. Science 1994, 266, 1013.

(3) Marlow, F.; McGehee, M. D.; Zhao, D.; Chmelka, B. F.; Stucky, G. D. Adv. Mater. 1999, 11, 632

(4) (a) Antonelli, D. M.; Ying, J. Y. Angew. Chem., Int. Ed. Engl. 1995, 34, 2015. (b) Mann, S.; Ozin, G. A. Nature 1996, 382, 313.

(5) Ying, J. Y.; Mehnert, C. P.; Wong, M. S. Angew. Chem., Int. Ed. Engl. 1999, 38, 56 .

(6) Stucky, G. D.; Hou, Q.; Firouzi, A.; Chemalka, B. F.; Schacht, S.; Voigt-Martin, I. G.; Schuth, F. Stud. Surf. Sci. Catal. 1997, 105, 3.

(7) Behrens, P. Angew. Chem. 1996, 108, 561.; Angew. Chem., Int. Ed. Engl. 1996, 35, 515.

(8) Zhao, X. S.; Lu, G. Q.; Millar, G. J. Ind. Eng. Chem. Res. 1996, 35, 2075.

(9) Diaz, J. F.; Balkus, J. K., Jr. J. Mol. Catal. 1996, 2, 115

(10) (a) Cheng, C. F.; Zhou, W. Z.; Klinowski, J. Chem. Phys. Lett. 1996, 263, 247. (b) Cheng, C. F.; Luan, Z.; Klinowski, J. Langmuir 1995, $11,2815$.

(11) Beck, J. S.; Vartuli, J. C.; Roth, W. J.; Leonowicz, M. E.; Kresge, C. T.; Schmitt, K. D.; Chu, C. T-W.; Olson, D. H.; Sheppard, E. W.; Higgins, S. B.; Schlenker, J. L. J. Am. Chem. Soc. 1992, 114, 10834.

(12) Chenite, A.; Page, Y. L.; Sayari, A. Chem. Mater. 1995, 7, 1015.

(13) Wu, C. N.; Tsai, T. S.; Liao, C. N.; Chao, K. J. Microporous Mater. 1996, 7,173 .

(14) Vartuli, J. C.; Schmitt, K. D.; Kresge, C. T.; Roth, W. J.; Leonowicz, M. E.; McCullen, S. B.; Helling, S. D.; Beck, J. S.; Schlenkeer, J. L.; Olsen, D. H.; Sheppard, E. W. Chem. Mater. 1994, 6, 2317.

(15) Tanev, P. T.; Liang, Y.; Pinnavaia, T. J. J. Am. Chem. Soc. 1997, 119,8616

(16) Kim, S. S.; Zhang, W.; Pinnavaia, T. J. Science 1998, 282, 1302.

(17) Tanev, P. T.; Pinnavaia, T. J. Science 1995, 267, 865.

(18) Zhang, W.; Froba, M.; Wang, J.; Tanev, P. T.; Wong, J.; Pinnavaia, T. J. J. Am. Chem. Soc. 1996, 118, 9164.

(19) Tanev, P. T.; Pinnavaia, T. J. Chem. Mater. 1996, 8, 2068

(20) Khushalani, D.; Kuperman, A.; Ozin, G. A.; Tanaka, K.; Garces, J.; Olken, M. M.; Kuperman, A. Adv. Mater. 1996, 7, 842.

(21) (a) Kim, J. M.; Kwak, J. H.; Jun, S.; Ryoo, R. J. Phys. Chem 1995, 99, 16742. (b) Ryoo, R.; Jun, S. J. Phys. Chem. B 1997, 101, 317.

(22) (a) Lin, H. P.; Cheng, S.; Mou, C.-Y. Microporous Mater. 1996 10, 111. (b) Lin, H. P.; Cheng, S.; Mou, C.-Y. J. Chin. Chem. Soc. 1996, 43,375 .

(23) Lin, H. P.; Mou, C.-Y. Science 1996, 273, 765. (b). Lin, H. P.; Cheng, Y. R.; Liu, S. B.; Mou, C.-Y. J. Mater. Chem. 1999, 9, 1197.

(24) (a) Lin, H. P.; Cheng, S.; Mou, C.-Y. Chem. Mater. 1998, 10, 581. (b) Cheng, Y. R.; Lin, H. P.; Mou, C.-Y. Phys. Chem. Chem. Phys. 1999, 1,5051 .

(25) Lin, H. P.; Wong, S. T.; Liu, S. B.; Mou, C.-Y.; Tang, C. Y. Stud. Surf. Sci. Catal. 2000, 129, 15 . 103.

(26) Wong, S. T.; Lin, H. P.; Mou, C. Y. Appl. Catal., A 2000, 198,

(27) Lin, H. P.; Mou, C.-Y. J. Cluster Sci. 1999, 10, 282.

(28) Sing, K. S. W.; Everett, D. H.; Haul, R. A. W.; Moscou, L.; Pirotti, R. A.; Roquerol, J.; Siemieniewska, T. Pure Appl. Chem. 1985, 57, 603. (29) Kruk, M.; Jaroniec, M.; Sakamoto, Y.; Terasaki, O.; Ryoo, R.; Ko, C. H. J. Phys. Chem. B 2000, 104, 292.

(30) (a) Kruk, M.; Jaroniec, M.; Sayari, A. Langmuir 1997, 13, 6267.

(b) Kruk, M.; Jaroniec, M.; Sayari, A. J. Phys. Chem. B 1997, 101, 583

(31) Lukens, W. W., Jr.; Schmidt-Winkel, P.; Zhao, D.; Feng, J.; Stucky, G. D. Langmuir 1999, 15, 5403 .

(32) Gregg, S. J.; Sing, K. S. W. Adsorption, Surface Area and Porosity; Academic Press: London, 1982

(33) Miyata, H.; Kuroda, K. Adv. Mater. 1999, 11, 875.

(34) (a) Chen, C.-Y.; Li, H.-X.; Davis, M. E. Microporous Mater. 1993, 2, 17. (b) Chen, C.-Y.; Burkett, S. L.; Li, H.-X.; Davis, M. E. Microporous Mater. 1993, 2, 27.

(35) (a) Reddy, K. M.; Song, C. Catal. Lett. 1996, 36, 103. (b) Kloetstra, K. R.; Zandbergen, H. W.; van Bekkum, H. Catal. Lett. 1995, 33, 157.

(36) Janicke, M. T.; Landry, C. C.; Christansen, S. C.; Birtanlan, S.; Stucky, G. D.; Chmelka, B. F. Chem. Mater. 1999, 11, 1342.

(37) Zhao, X. S.; Lu, G. Q.; Hu, X. Chem. Commun. 1999, 1391.

(38) Schmidt-Winkel, P.; Lukens, W. W., Jr.; Zhao, D.; Yang, P.; Chmelka, F.; Stucky, G. D. J. Am. Chem. Soc. 1999, 121, 254. 
(39) (a) Kruk, M.; Jaroniec, M. Chem. Mater. 2000, 12, 222. (b) Kruk, M.; Jaroniec, M., Sayari, A.; Chem. Mater. 1999, 11, 492. (c) Sayari, A.; Yang, Y.; Kruk, M.; Jaroniec, M. J. Phys. Chem. B 1999, 103, 3651.

(40) Long, Y.; Xu, T.; Sun, Y.; Dong, W. Langmuir 1998, 14, 6173.

(41) Everett, D. H. In The Solid-Gas Interface; Flood, E. A., Ed.; Marcel Dekker: New York, '967; Vol. 2, pp 1055-1113.
(42) (a) Mayagoitia, V. J. Chem. Soc., Faraday Trans. 1985, 81, 2931. (b) Mayagoitia, V.; Rojas, F.; Kornhauser, I. J. Chem. Soc., Faraday Trans. 1988, 84, 785 (c) Mayagoitia, V.; Gilot, B.; Rojas, F.; Kornhauser, I. J. Chem. Soc., Faraday Trans. 1988, 84, 801.

(43) Edler, K. J.; Reynolds, P. A.; Branton, P. J.; Trouw, F. R.; White, J. W. J. Chem. Soc., Faraday Trans. 1997, 93, 1667. 